\title{
Experimental and DFT mechanistic study of dehydrohalogenation of 1-chloro-1,1-difluoroethane over metal fluorides
}

Wenfeng Han, ${ }^{\text {*a }}$ Bing Liu, ${ }^{\text {a }}$ Yikun Kang, ${ }^{\mathrm{b}}$ Zhikun Wang, ${ }^{\text {a }}$ Wei Yu, ${ }^{\mathrm{a}}$ Hong Yang, Yongnan Liu, ${ }^{\mathrm{a}}$ Jiaqin Lu, ${ }^{\text {a }}$ Haodong Tang, ${ }^{\mathrm{a}}$ Ying Li, ${ }^{\mathrm{a}}$ Weiyu Song ${ }^{* \mathrm{~b}}$

a Institute of Industrial Catalysis, Zhejiang University of Technology, Chaowang Road 18, Hangzhou 310014, Zhejiang, P. R. China.

b State Key Laboratory of Heavy Oil Processing, College of Science, China University of Petroleum, Beijing, 18 Fuxue Road, Beijing, 102249, China

*E-mail: hanwf@zjut.edu.cn (W.H.)

songwy@cup.edu.cn (W. S.)

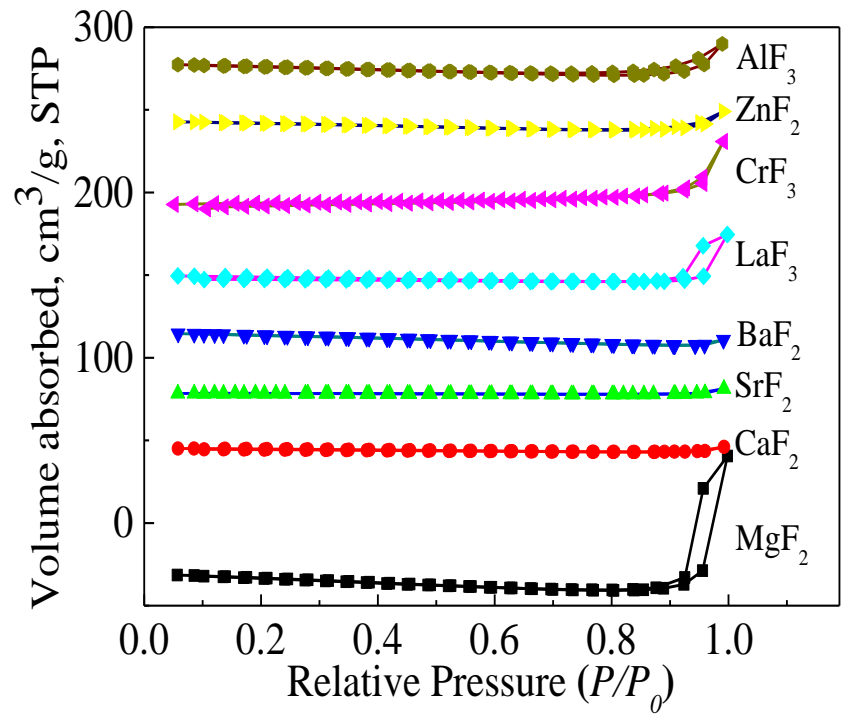

Figure $\mathrm{S} 1 . \mathrm{N}_{2}$ adsorption isotherms of all metal fluoride catalysts. 
Table S1. Surface areas and pore volumes of fresh metal fluoride catalysts determined by the BET equation.

\begin{tabular}{ccc}
\hline Samples & $\mathrm{S}_{\mathrm{BET}}, \mathrm{m}^{2} / \mathrm{g}$ & $\mathrm{V}, \mathrm{cm}^{3} / \mathrm{g}$ \\
\hline $\mathrm{KF}$ & -- & -- \\
$\mathrm{MgF}_{2}$ & 1.3 & 0.11 \\
$\mathrm{CaF}_{2}$ & 0.1 & 0.002 \\
$\mathrm{SrF}_{2}$ & 1.4 & 0.005 \\
$\mathrm{BaF}_{2}$ & -- & -- \\
$\mathrm{LaF}_{3}$ & 0.4 & 0.04 \\
$\mathrm{CrF}_{3}$ & 11.1 & 0.06 \\
$\mathrm{ZnF}_{2}$ & 0.2 & 0.01 \\
$\mathrm{AlF}_{3}$ & 0.2 & 0.02 \\
\hline
\end{tabular}

Table S2. Reaction enthalpy of DeF $\left(E_{R}-D e F\right)$ and $\mathrm{DeCl}\left(\mathrm{E}_{\mathrm{R}}-\mathrm{DeCl}\right)$ on different metal fluorides.

\begin{tabular}{cccccc}
\hline $\begin{array}{c}\text { Metal } \\
\text { fluorides }\end{array}$ & $\mathrm{KF}$ & $\mathrm{CaF}_{2}$ & $\mathrm{SrF}_{2}$ & $\mathrm{BaF}_{2}$ & $\mathrm{AlF}_{3}$ \\
\hline $\begin{array}{c}\mathrm{E}_{\mathrm{R}}-\mathrm{DeF} \\
(\mathrm{eV})\end{array}$ & 4.39 & 4.26 & 4.23 & 4.16 & 3.15 \\
$\begin{array}{c}\mathrm{E}-\mathrm{DeCl} \\
(\mathrm{eV})\end{array}$ & 3.41 & 3.28 & 3.20 & 3.10 & 3.50 \\
\hline
\end{tabular}


Table S3. The peak area of $\mathrm{NH}_{3}$-TPD obtained by integral and the peak area ratio with $\mathrm{KF}$ as reference.

\begin{tabular}{ccc}
\hline Catalysts & Peak area & The ratio $(\mathrm{KF}=1)$ \\
\hline $\mathrm{KF}$ & 15.4 & 1.0 \\
$\mathrm{MgF}_{2}$ & 423.8 & 27.5 \\
$\mathrm{CaF}_{2}$ & 108.8 & 7.1 \\
$\mathrm{SrF}_{2}$ & 196.8 & 12.8 \\
$\mathrm{BaF}_{2}$ & 158.8 & 10.3 \\
$\mathrm{LaF}_{3}$ & 114.7 & 7.4 \\
$\mathrm{CrF}_{3}$ & 242.5 & 15.7 \\
$\mathrm{ZnF}_{2}$ & 24.0 & 1.56 \\
$\mathrm{AlF}_{3}$ & 411.9 & 26.7 \\
\hline
\end{tabular}

Table S4. Gibbs free energy changes of reactions between metal fluorides and $\mathrm{HCl}$ at $327{ }^{\circ} \mathrm{C}$.

\begin{tabular}{cccc}
\hline Metal & $\begin{array}{c}\triangle_{\mathrm{f}} G \text { of chloride, } \\
\mathrm{kJ} / \mathrm{mol}\end{array}$ & $\begin{array}{c}\triangle_{\mathrm{f}} G \text { of fluoride, } \\
\mathrm{kJ} / \mathrm{mol}\end{array}$ & $\begin{array}{c}\Delta \mathrm{r} G, \mathrm{~kJ} / \mathrm{mol} \\
\mathrm{MFx}+\mathrm{xHCl} \rightarrow \mathrm{MClx}+\mathrm{xHF}\end{array}$ \\
\hline $\mathrm{K}$ & -379.09 & -507.441 & -50.25 \\
$\mathrm{Mg}$ & -543.56 & -1018.32 & 117.57 \\
$\mathrm{Ca}$ & -701.47 & -1122.18 & 63.52 \\
$\mathrm{Sr}$ & -732.23 & -1112.84 & 23.42 \\
$\mathrm{Ba}$ & -762.47 & -1108.57 & -11.08 \\
$\mathrm{La}$ & -921.37 & -1548.91 & 91.76 \\
$\mathrm{Cr}$ & -417.59 & -1034.26 & 80.89 \\
$\mathrm{Zn} *$ & -324.79 & -663.302 & -18.68 \\
$\mathrm{Al} *$ & -555.18 & -1351.94 & 260.98 \\
\hline$\triangle$
\end{tabular}

$\triangle_{\mathrm{f}} G(\mathrm{HF})=-276.57 \mathrm{~kJ} / \mathrm{mol} \quad \triangle_{\mathrm{f}} G(\mathrm{HCl})=-97.975 \mathrm{~kJ} / \mathrm{mol}$

* At $327{ }^{\circ} \mathrm{C}, \mathrm{ZnCl}_{2}$ is in form of liquid (melting point of about $283 \sim 293{ }^{\circ} \mathrm{C}$ ), and $\mathrm{AlCl}_{3}$ is in the form of gasphase (melting point of around $181^{\circ} \mathrm{C}$ ). 
Table S5. The reaction enthalpy of adsorption of $\mathrm{F}$ ( $\left.\mathrm{E}_{\mathrm{Fads}}\right)$ or $\mathrm{Cl}$ ( $\left.\mathrm{E}_{\text {Clads }}\right)$ radicals

\begin{tabular}{cccccc}
\hline $\begin{array}{c}\text { Metal } \\
\text { fluorides }\end{array}$ & $\mathrm{KF}$ & $\mathrm{CaF}_{2}$ & $\mathrm{SrF}_{2}$ & $\mathrm{BaF}_{2}$ & $\mathrm{AlF}_{3}$ \\
\hline $\mathrm{E}_{\text {Fads }}(\mathrm{eV})$ & -1.06 & -1.20 & -1.23 & -1.30 & -2.30 \\
$\mathrm{E}_{\text {Clads }}(\mathrm{eV})$ & -0.41 & -0.54 & -0.62 & -0.72 & -0.33 \\
\hline
\end{tabular}

Table S6. The difference between $\mathrm{E}_{\text {Fads }}$ and $\mathrm{E}_{\text {Clads }}$ for each metal fluoride.

\begin{tabular}{cccccc}
\hline $\begin{array}{c}\text { Metal } \\
\text { fluorides }\end{array}$ & $\mathrm{KF}$ & $\mathrm{CaF}_{2}$ & $\mathrm{SrF}_{2}$ & $\mathrm{BaF}_{2}$ & $\mathrm{AlF}_{3}$ \\
\hline $\begin{array}{c}\text { E } \\
\begin{array}{c}\text { Clads }-\mathrm{E}_{\text {Fads }} \\
(\mathrm{eV})\end{array}\end{array}$ & 0.65 & 0.66 & 0.61 & 0.58 & 1.98 \\
\hline
\end{tabular}

Table S7 The surface elemental content of $\mathrm{SrF}_{2}$ catalyst following different reaction time determined by EDS.

\begin{tabular}{ccccccc}
\hline Sample & \multicolumn{5}{c}{ Elemental content, mol\% } & \multirow{2}{*}{$\mathrm{F} / \mathrm{Cl}$} \\
\cline { 2 - 5 } & $\mathrm{C}$ & $\mathrm{O}$ & $\mathrm{F}$ & $\mathrm{Cl}$ & $\mathrm{Sr}$ & \\
\hline $\mathrm{SrF}_{2}-\mathrm{Ch}$ & 0 & 5.77 & 60.09 & 0 & 34.14 & 0 \\
$\mathrm{SrF}_{2}-2 \mathrm{~h}$ & 26.03 & 2.31 & 27.25 & 18.64 & 25.77 & 0.68 \\
$\mathrm{SrF}_{2}-4 \mathrm{~h}$ & 28.13 & 2.18 & 21.95 & 22.27 & 25.48 & 0.89 \\
$\mathrm{SrF}_{2}-6 \mathrm{~h}$ & 30.12 & 2.22 & 22.54 & 20.96 & 24.16 & 0.94 \\
$\mathrm{SrF}_{2}-8 \mathrm{~h}$ & 32.33 & 1.86 & 21.63 & 20.37 & 23.82 & 0.95 \\
\hline
\end{tabular}



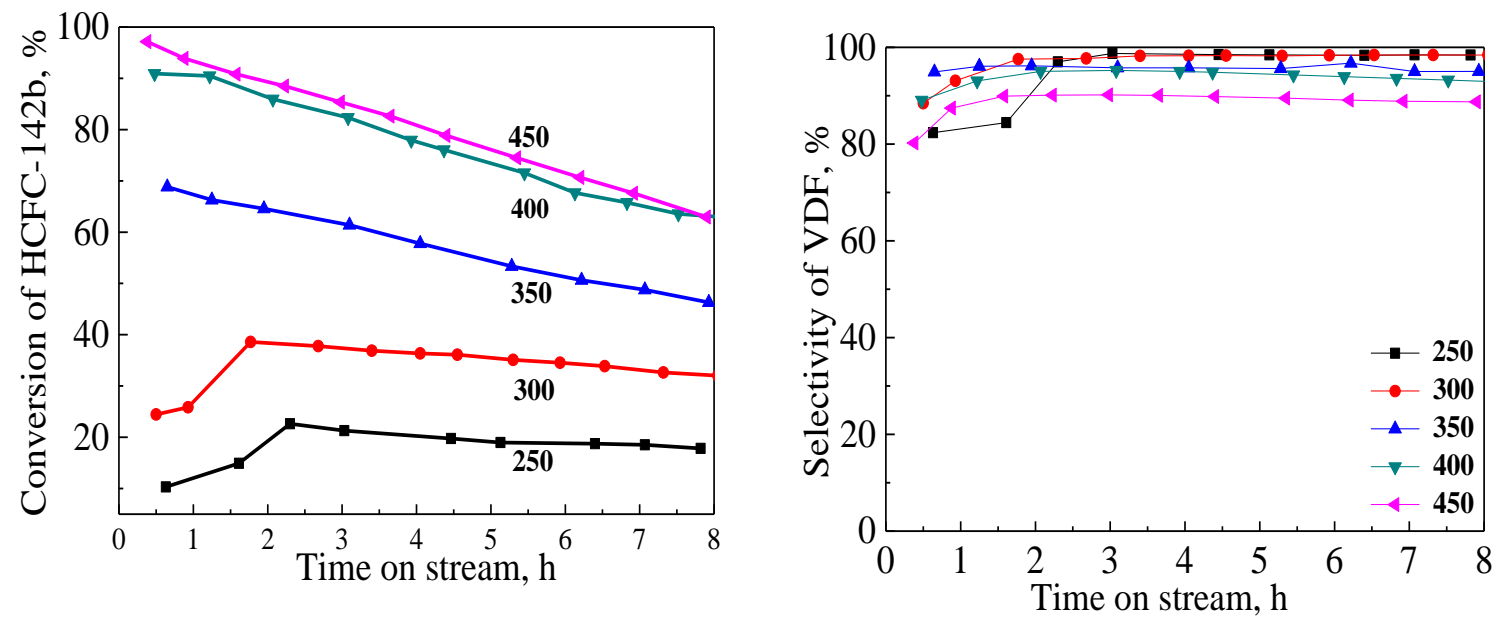

Figure S2. Effect of reaction temperature on the stability of $\mathrm{BaF}_{2}$ catalyst for the pyrolysis of HCFC-142b to vinylidene fluoride. Reaction conditions: 1 bar, $\mathrm{N}_{2}: \mathrm{HCFC}-$ $142 \mathrm{~b}$ of $1: 1$, GHSV(HCFC-142b) of $600 \mathrm{~h}^{-1}$.

Table S8 Gibbs Free Energy of reaction between $\mathrm{BaF}_{2}$ or $\mathrm{SrF}_{2}$ and $\mathrm{HCl}$ at elevated temperatures.

\begin{tabular}{ccccc}
\hline Metal & $T,{ }^{\circ} \mathrm{C}$ & $\begin{array}{c}\triangle_{\mathrm{f}} G \text { of chloride, } \\
\mathrm{kJ} / \mathrm{mol}\end{array}$ & $\begin{array}{c}\triangle_{\mathrm{f}} G \text { of fluoride, } \\
\mathrm{kJ} / \mathrm{mol}\end{array}$ & $\begin{array}{c}\Delta \mathrm{r} G, \mathrm{~kJ} / \mathrm{mol} \\
\mathrm{MF}_{2}+2 \mathrm{HCl} \rightarrow \mathrm{MCl}_{2}+2 \mathrm{HF}\end{array}$ \\
\hline $\mathrm{Ba}$ & 227 & -778.24 & -1125.00 & -10.44 \\
& 327 & -762.47 & -1108.57 & -11.08 \\
& 427 & -746.70 & -1092.18 & -11.70 \\
& 527 & -731.00 & -1075.88 & -12.31 \\
\hline $\mathrm{Sr}$ & 227 & -747.71 & -1129.68 & 24.78 \\
& 327 & -732.23 & -1112.84 & 23.42 \\
& 427 & -717.018 & -1096.19 & 21.99 \\
& 527 & -702.01 & -1079.68 & 20.49 \\
\hline
\end{tabular}


Table S9. The reaction enthalpy of $\mathrm{DeF}\left(\mathrm{E}_{\mathrm{R}}-\mathrm{DeF}\right)$ and $\mathrm{DeCl}\left(\mathrm{E}_{\mathrm{R}}-\mathrm{DeCl}\right)$ on $\mathrm{AlF}_{3}(111)$ with different proportions of $\mathrm{F}$ defects.

\begin{tabular}{ccccccc}
\hline F defects & $1 / 14 \mathrm{ML}$ & $1 / 7 \mathrm{ML}$ & $3 / 14 \mathrm{ML}$ & $2 / 7 \mathrm{ML}$ & $5 / 14 \mathrm{ML}$ & $3 / 7 \mathrm{ML}$ \\
\hline $\begin{array}{c}\mathrm{E}_{\mathrm{R}}-\mathrm{DeF} \\
(\mathrm{eV})\end{array}$ & 3.91 & 3.84 & 3.47 & 3.70 & 3.58 & 3.15 \\
$\begin{array}{c}\mathrm{E}_{\mathrm{R}}-\mathrm{DeCl} \\
(\mathrm{eV})\end{array}$ & 2.55 & 3.37 & 3.52 & 3.67 & 3.74 & 3.50 \\
\hline
\end{tabular}

Table S10. The difference between $\mathrm{E}_{\text {Fads }}$ and $\mathrm{E}_{\text {Clads }}$ on $\mathrm{AlF}_{3}(111)$ with different proportions of $\mathrm{F}$ defects.

\begin{tabular}{ccccccc}
\hline F defects & $1 / 14 \mathrm{ML}$ & $1 / 7 \mathrm{ML}$ & $3 / 14 \mathrm{ML}$ & $2 / 7 \mathrm{ML}$ & $5 / 14 \mathrm{ML}$ & $3 / 7 \mathrm{ML}$ \\
\hline $\begin{array}{c}\mathrm{E}_{\text {Fads }}- \\
\mathrm{E}_{\text {Clads }}(\mathrm{eV})\end{array}$ & 0.27 & 1.16 & 1.68 & 1.61 & 1.80 & 1.98 \\
\hline
\end{tabular}

